\title{
Fully endoscopic combined transsphenoidal and supraorbital keyhole approach for parasellar lesions
}

\author{
Yuichi Nagata, MD, ${ }^{1,2}$ Tadashi Watanabe, MD, PhD, ${ }^{2}$ Tetsuya Nagatani, MD, PhD, ${ }^{2}$ \\ Kazuhito Takeuchi, MD, ${ }^{1}$ Jonsu Chu, MD, ${ }^{1}$ and Toshihiko Wakabayashi, MD, PhD ${ }^{1}$ \\ 1Department of Neurosurgery, Nagoya University Graduate School of Medicine; and 2Department of Neurosurgery, Japanese \\ Red Cross Nagoya Daini Hospital, Nagoya, Aichi, Japan
}

\begin{abstract}
OBJECTIVE Parasellar tumors that extend far laterally beyond the internal carotid artery or that are fibrous and adhere firmly to critical structures are difficult to remove totally via the endoscopic transsphenoidal approach alone. In such cases, a combined transsphenoidal-transcranial approach is effective to achieve maximal resection in a single stage. In this paper, a new minimally invasive surgical technique for complicated parasellar lesions, a fully endoscopic combined transsphenoidal-supraorbital keyhole approach, is presented.
\end{abstract}

METHODS A retrospective review of patients who had been treated via a fully endoscopic combined transsphenoidalsupraorbital keyhole approach for complicated parasellar lesions was performed. The data for resection rate, perioperative mortality and morbidity, and postoperative outcomes were analyzed.

RESULTS A total of 12 fully endoscopic combined transsphenoidal-supraorbital keyhole approaches were performed from March 2013 to February 2016; 10 were for pituitary adenomas and 2 were for craniopharyngiomas. Gross-total resection or near-total resection was achieved in 7 of 12 cases. Among the 11 patients who had presented with preoperative visual disturbances, 7 had visual improvement. However, 1 patient showed deterioration in visual function. No patient experienced postoperative hemorrhage, needed additional surgical treatment, or had postoperative CSF leakage.

CONCLUSIONS In the combined transsphenoidal and transcranial approach, safe and effective cooperative manipulation with 2 surgical corridors can be performed for complicated parasellar lesions. The goal of this procedure is not to achieve gross-total resection, but to achieve safe resection. Moreover, this new surgical approach offers neurosurgeons a simpler operative field with less invasiveness than the conventional microscopic combined approach. The fully endoscopic combined endonasal-supraorbital keyhole approach is an efficacious procedure for complicated parasellar lesions with acceptable results.

https://thejns.org/doi/abs/10.3171/2016.11.JNS161833

KEY WORDS combined approach; transsphenoidal approach; supraorbital approach; pituitary adenoma; craniopharyngioma; keyhole; oncology

$\mathrm{T}$ HE endoscopic transsphenoidal approach (eTSA) for parasellar lesions has become a standard neurosurgical procedure over the last decade. Many pituitary adenomas and other tumors arising at the parasellar region can be safely and completely resected via this approach. $3,4,6-8,10,12,13,16,17,31,37,39$ However, some cases, which are fibrous, tightly adherent to critical structures, or extend far laterally beyond the internal carotid artery (ICA), are difficult to remove totally via this approach alone, or even through the transcranial approach (TCA) alone. A firm and/or vascularized residual pituitary adenoma frequently causes postoperative hemorrhage, resulting in severe surgical morbidity and mortality. ${ }^{12,29,39}$ In addition, residual craniopharyngiomas that adhere firmly to adjacent neurovascular structures often regrow rapidly after the surgery. However, aggressive resection of adherent tumor can cause damage to surrounding critical structures, also resulting in severe surgical complications. Various surgical procedures have been reported for this situation, $3,28,29,32$ and simultaneous combined TSA-TCA is an effective surgical option to

ABBREVIATIONS ACTH = adrenocorticotropic hormone; CSDH = chronic subdural hematoma; $\mathrm{DI}$ = diabetes insipidus; eSKA = endoscopic SKA; eTSA = endoscopic TSA; $\mathrm{FSH}=$ follicle-stimulating hormone; $\mathrm{GH}$ = growth hormone; GTR = gross-total resection; ICA = internal carotid artery; LH = luteinizing hormone; NFPA = nonfunctional pituitary adenoma; NTR = near-total resection; SKA = supraorbital keyhole approach; TCA = transcranial approach; TSA = transsphenoidal approach; TSH = thyroid-stimulating hormone.

SUBMITTED July 25, 2016. ACCEPTED November 21, 2016.

INCLUDE WHEN CITING Published online April 28, 2017; DOI: 10.3171/2016.11.JNS161833. 


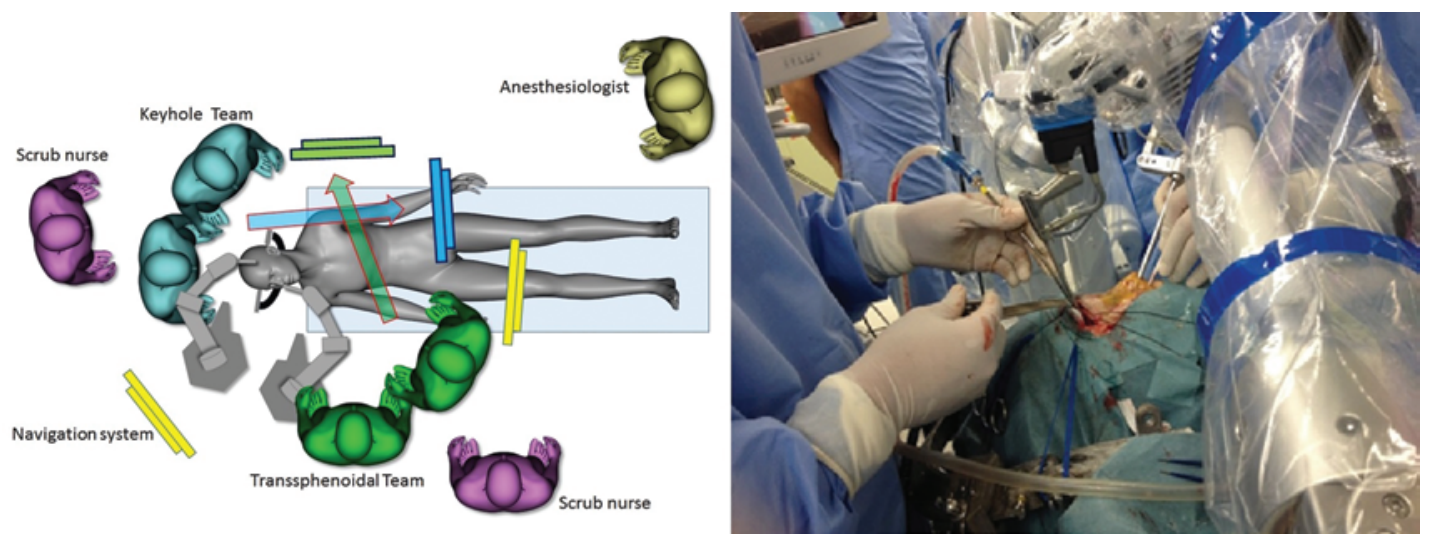

FIG. 1. The schema of our operating room setup for the fully endoscopic TSA and SKA. Two operative fields, 2 neurosurgical teams, and 2 neuroendoscopic systems with 2 monitors are set up around the patient (left). The eTSA and eSKA are performed simultaneously under a simple surgical field without interfering with the other surgical team (right). Figure is available in color online only.

optimize resection of these complicated tumors in a single stage. $1,5,11,15,18,19,21,23,24,27$ One of the greatest advantages of a simultaneous combined TSA-TCA is the cooperation from 2 surgical directions, leading to greater safety during tumor resection. In fact, several types of combinations have been reported: microscopic TSA-microscopic TCA, $, 1,5,18,21,23$ eTSA-microscopic TCA, ${ }^{15,19}$ microscopic TSA-endoscopic transventricular approach, ${ }^{24}$ and eTSAendoscopic transventricular approach. ${ }^{11,27}$ The operative field of the traditional combined approach, however, seems complicated. Moreover, the traditional combined approach is highly invasive for patients. In this paper, we present a new combined approach involving a fully endoscopic combined TSA and supraorbital keyhole approach (SKA; a type of TCA), which is simpler and less invasive than the conventional microscopic combined approach.

\section{Methods}

After receiving approval from the Japanese Red Cross Nagoya Daini Hospital's institutional review board, we performed a retrospective review of records of patients who had been treated by a fully endoscopic combined TSA-SKA for complicated parasellar lesions.

\section{Patient Selection}

Fully endoscopic combined TSA-SKA was indicated only for a select group of patients with complicated parasellar lesions that 1) extended laterally beyond the lateral edge of ICA; and/or 2) were fibrous and adhered firmly to adjunct critical neurovascular structures, including the ICA and its branches, optic apparatus, cranial nerves, and brain parenchyma. Parasellar lesions with this configuration and consistency are difficult to manage by TSA alone, even with the extended skull base technique. Moreover, tumor consistency and strong adhesion to the surrounding critical structures are not accurately predicted by preoperative images alone. Therefore, most combined procedures were performed for recurrent tumors that had resulted from partial resection during previous surgery due to such fibrous tumor consistency. The exclusion criteria for this approach were parasellar lesions with excessive invagi- nation to the frontal lobe, those with bilateral extension beyond the ICAs, and/or those with excessive progression into the posterior fossa.

\section{Preoperative Assessment}

All patients underwent routine evaluation for tumor size and configuration with preoperative MRI, and surrounding vascular information was also assessed by preoperative 3D CT angiography. Basal hormonal status was assessed in all patients, and dynamic hormonal status was also assessed when clinically appropriate. Visual disturbance was evaluated by visual acuity examination and visual field examination.

\section{Surgical Procedure}

This approach involves the eTSA and the endoscopic supraorbital keyhole approach (eSKA) and requires 2 operative fields, 2 neurosurgical teams, and 2 neuroendoscopic systems with 2 monitors. The neuroendoscopic system is smaller than the microscopic system, providing a much more comfortable working space without interfering with the other surgical team (Fig. 1). The 2 neurosurgical teams included 2 operative surgeons (the eTSA operator and the eSKA operator), and their assistants. A third surgeon, however, is the key person for effective cooperative manipulation in simultaneous combined eTSA-eSKA. This surgeon always watches the monitors for both approaches and understands the entire context of the surgery, providing the operating surgeons with precise instructions depending on the surgical situation. Usually, the most senior surgeon acts as the third surgeon.

After induction of general anesthesia using endotracheal intubation, the patient was positioned supine, with the upper body raised $15^{\circ}$. The head was fixed in a Sugita 4-point head holder (Mizuho Medical Innovation) and rotated $10^{\circ}-20^{\circ}$ to the contralateral side of the SKA. The side of the SKA was determined according to the predominant direction of tumor extension. After all operative fields, including forehead, eyebrow, nose, and abdomen, were prepared with a povidone-iodine solution, eTSA and eSKA were performed simultaneously. 


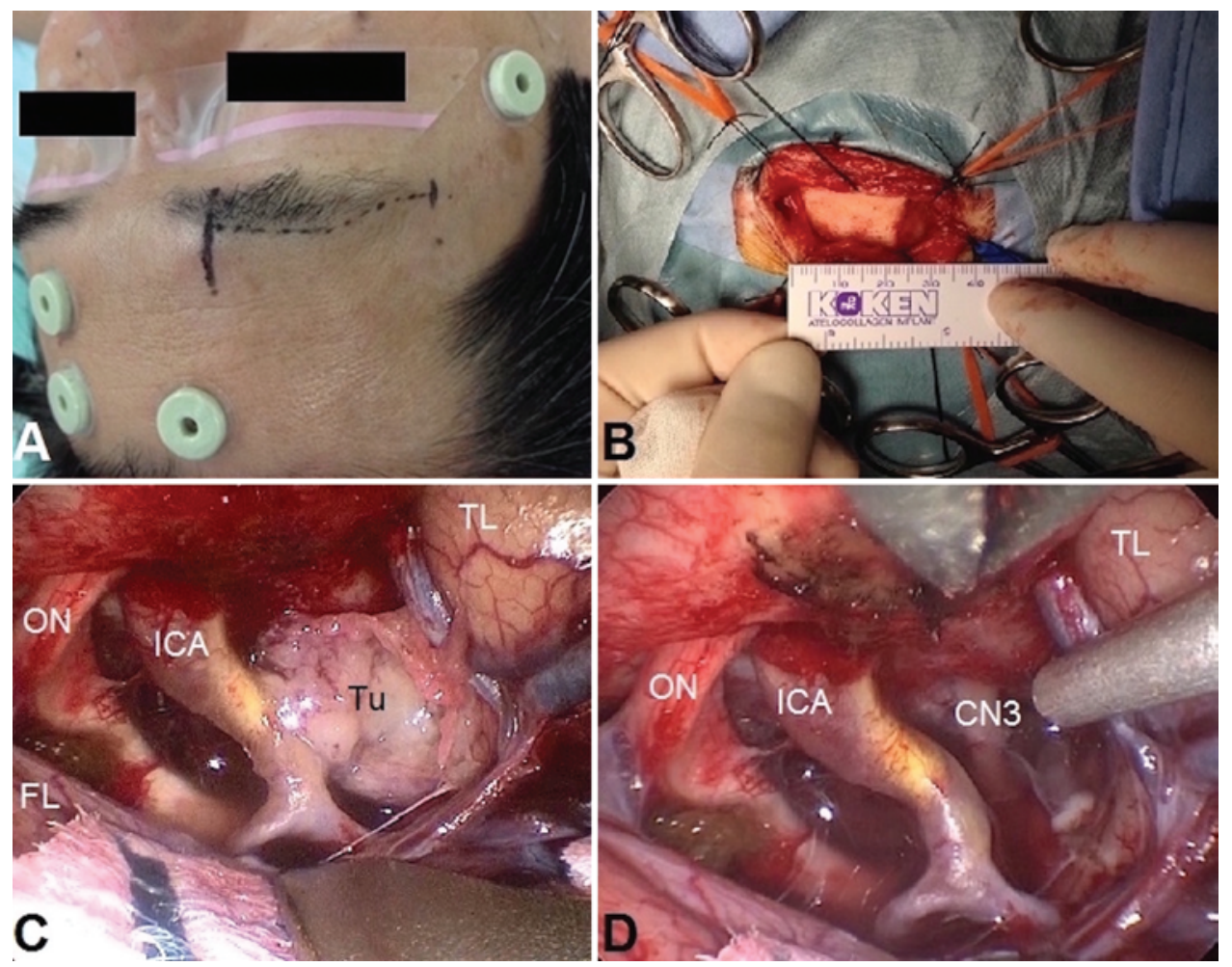

FIG. 2. Intraoperative images obtained during the eSKA. A: The skin incision is made along the superior edge of the eyebrow. B: After dissection of the frontal and temporal muscles, the craniotomy, with a size of approximately $2.5 \times 1.5 \mathrm{~cm}$, can be performed. C: After adequate CSF drainage, tumor resection is performed under visualization through the rigid endoscope. Tumor extending far laterally beyond the ICA and the surrounding neurovascular structures is well exposed via this approach with minimal retraction by the brain spatula. D: The final view after the tumor resection. A wide operative field from the anterior skull base to the parasellar region is available in this approach. $\mathrm{CN} 3=$ oculomotor nerve; $\mathrm{FL}=$ frontal lobe; $\mathrm{ON}=$ optic nerve; $\mathrm{TL}=$ temporal lobe; Tu = tumor. Figure is available in color online only.

\section{Endoscopic Transsphenoidal Approach}

Using a rigid endoscope with a pneumatic holder, a vertical incision was made on the nasal septal mucosa. Submucosal dissection was performed, and septal bone was removed. Through the submucosal corridor, the sphenoid sinus could be reached with minimal injury to intranasal structures. Wide sphenoidotomy is the key point of this procedure to obtain appropriate operative exposure; thus, a sufficient amount of the anterior wall and septum of the sphenoid sinus must be removed. The sellar floor was then opened widely to expose the dura mater rostrally to the tuberculum sellae and the cavernous sinuses bilaterally. Subsequently, the dura mater was opened depending on the shape of the tumor. If needed, anterior extension of the dural incision with removal of the tuberculum sellae and planum sphenoidale was performed. After dissecting the anterior part of the tumor capsule from the dura mater, the intrasellar part of the tumor was debulked. Then, the border between the tumor and pituitary gland is identified, and dissection is continued along this plane. The removal is continued in the suprasellar part of the tumor with careful manipulation. With a large and multilobular tumor, the view of the superior and posterior aspects of the tumor, where adhesion between the tumor and the surrounding critical neurovascular structures may exist, is often obscured. An attempt to remove the tumor forcibly via eTSA may cause irreversible damage to the adjacent neurovascular structures, leading to perioperative intracranial hemorrhage or a permanent neurological deficit. In this situation, cooperative manipulation with the eSKA team is quite useful. The eSKA surgeon is able to identify these critical points better than the eTSA surgeon. After the tumor was separated from all adjacent critical structures by the eSKA, the suprasellar part of the tumor was finally removed by the eTSA.

After removal of the tumor, a direct communication exists between the subarachnoid space and/or the third ventricle and the sphenoid sinus. Watertight closure of the dura mater, therefore, is quite important to prevent postoperative CSF leakage. The method of dural closure was described in a previous report. ${ }^{33}$ The intrasellar space was filled with free fat graft harvested from the abdomen, and the dura mater is sutured in a watertight manner with 6-0 Prolene sutures. If high-flow CSF leakage were detected intraoperatively, a fascia graft was additionally placed as inlay and sutured to dura mater in a watertight manner. Dural sealant was then sprayed to ensure prevention of postoperative CSF leakage. A spinal drain was not placed.

\section{Endoscopic Supraorbital Keyhole Approach}

The skin incision was made along the superior edge of the eyebrow, avoiding damage to the supraorbital nerve (Fig. 2A). After subcutaneous dissection of the skin flap, the frontal muscle was incised in a linear fashion. The 
TABLE 1. Clinical characteristics of all 12 patients

\begin{tabular}{|c|c|c|c|c|c|c|}
\hline \multirow{2}{*}{$\begin{array}{l}\text { Case } \\
\text { No. }\end{array}$} & \multirow{2}{*}{$\begin{array}{c}\text { Age (yrs) } \\
\text { Sex }\end{array}$} & \multirow[b]{2}{*}{ Pathology } & \multirow{2}{*}{$\begin{array}{l}\text { Max Diameter } \\
(\mathrm{mm})\end{array}$} & \multicolumn{2}{|l|}{ Preop Presentation } & \multirow[b]{2}{*}{ Previous Intervention } \\
\hline & & & & Visual Disturbance & HPD & \\
\hline 1 & $78, M$ & Rec NFPA & 62 & Bilat hemianopia, It DVA, OMD & None & TSA \\
\hline 2 & $28, F$ & Rec NFPA & 24.8 & Unilat hemianopia, rt DVA & Partial & Extended TSA \\
\hline 3 & $68, F$ & Rec NFPA & 53.3 & Bilat hemianopia, It DVA & $\mathrm{PH}$ & TSA $\times 4$ \\
\hline 4 & $79, \mathrm{M}$ & Rec NFPA & 43.8 & Bilat hemianopia & Partial & $\mathrm{TSA} \times 2$ \\
\hline 5 & $25, M$ & Rec NFPA & 27.8 & Bilat hemianopia & Partial & TSA, combined TSA \& TCA \\
\hline 6 & $41, \mathrm{M}$ & Rec NFPA & 37 & $\mathrm{Rt} \mathrm{HH}$ & None & TSA \\
\hline 7 & $27, M$ & Rec NFPA & 43.3 & Bilat hemianopia, rt DVA & None & TSA \\
\hline 8 & $46, M$ & NFPA & 44 & Unilat quadrantanopia & None & None \\
\hline 9 & $32, F$ & Rec craniopharyngioma & 33.8 & None & $\mathrm{PH}, \mathrm{DI}$ & TCA, extended TSA, SRS \\
\hline 10 & $68, M$ & NFPA & 29.8 & Lt DVA & None & None \\
\hline 11 & $63, M$ & NFPA & 38.9 & Lt HH & None & None \\
\hline 12 & $38, M$ & Craniopharyngioma & 45.2 & Unilat hemianopia, It DVA & Partial & None \\
\hline
\end{tabular}

$\mathrm{DVA}=$ decreased visual acuity; $\mathrm{HH}=$ homonymous hemianopia; $\mathrm{HPD}=$ hormonal pituitary dysfunction; $\mathrm{OMD}=$ ocular motility disorder; partial = partial hypopituitarism (deficiencies in 2 or 3 axes); $\mathrm{PH}=$ panhypopituitarism (deficiencies in $\geq 3$ axes); Rec = recurrent; SRS = stereotactic radiosurgery.

temporal muscle was detached from the superior temporal line and retracted laterally to expose the keyhole area. The craniotomy, with a size of approximately $2.5 \times 1.5$ $\mathrm{cm}$, was performed with a bur hole at the keyhole area (Fig. 2B). The dura mater was opened in a semiarc fashion and reflected inferiorly. Subsequent manipulation was performed under visualization of the rigid endoscope with a pneumatic holder. We prefer a 4-mm outer diameter endoscope because it provides much higher quality images than smaller endoscopes. A pneumatic holder is an essential surgical instrument for intracranial manipulation under a rigid endoscope to prevent accidental injury of the surrounding critical neurovascular structures. Under visualization of the rigid endoscope, CSF was first drained. Opening of the sylvian fissure can also facilitate CSF drainage. Sufficient CSF drainage allows lifting of the frontal lobe gravitationally with minimal retraction force. As needed, a 5-mm nontapering brain spatula was used, which minimizes interference with the surgical instruments. The wide operative field from the anterior skull base to the parasellar region was exposed through this technique. After identifying the tumor and the surrounding critical structures, tumor resection was started (Fig. 2C). This approach is suitable to remove the tumor located far laterally beyond the ICA, where it is limited by the eTSA. Subsequently, the suprasellar part of the tumor was dissected from the adjacent critical neurovascular structures, including the optic apparatus, the ICA, and its branches, and the surrounding brain parenchyma, while preserving the arachnoid planes to assist tumor removal by the eTSA. If tumor adhesion to the adjacent critical structures is too difficult to dissect, the eSKA operator may leave a small amount of tumor at the point of solid adhesion to prevent surgical complications.

After tumor resection (Fig. 2D), the eSKA team closed the dura mater in a watertight manner with 4-0 Prolene suture, and dural sealant was also sprayed. The bone flap was fixed with titanium plates. Bionic bone paste was used to relieve postoperative deformation. After the skin was closed in each layer, the incision was covered with topical skin adhesive.

\section{Results}

Between March 2013 and February 2016, 12 fully endoscopic combined TSA-SKA procedures were performed in patients with complicated parasellar lesions. There were 9 men and 3 women with a mean age of 49.4 years (range 25-79 years). On histopathological examination of the tumors, there were 10 pituitary adenomas and 2 craniopharyngiomas, of which 7 pituitary adenomas and 1 craniopharyngioma were recurrent tumors. All pituitary adenomas were clinically nonfunctional pituitary adenomas (NFPAs). The average tumor size was $40.3 \mathrm{~mm}$ (range 24.8-62 mm). Preoperative symptoms included visual disturbance in 11 patients (91.7\%), ocular motility disturbance in 1 patient (8.3\%), anterior hormonal pituitary dysfunction in 5 patients (41.7\%), and diabetes insipidus (DI) in 1 patient (8.3\%). Patient demographics, tumor pathologies, preoperative signs and symptoms, and previous interventions are described in Table 1.

\section{Postoperative Outcomes}

Gross-total resection (GTR) and near-total resection (NTR) $(\geq 95 \%)$ were achieved in 7 of 12 cases (58.3\%). The extent of resection was not assessed based on intraoperative findings alone but was also confirmed on MRI 1 week after the surgery. Any residual tumor capsule was classified as an NTR. Of the 11 patients who had presented with preoperative visual disturbances, 7 (63.6\%) had visual improvement. However, 1 patient experienced deterioration in visual function: the preoperative partial unilateral hemianopia in her right eye deteriorated, with additional upper altitudinal hemianopia postoperatively, but there was no change in visual acuity.

With regard to postoperative morbidity, 3 patients developed anterior hormonal pituitary deterioration: in Case 3 , deficiencies in 3 axes (growth hormone [GH], thyroid- 
TABLE 2. Surgical outcomes of all 12 patients

\begin{tabular}{|c|c|c|c|c|c|c|c|}
\hline \multirow{2}{*}{$\begin{array}{l}\text { Case } \\
\text { No. }\end{array}$} & \multirow{2}{*}{$\begin{array}{l}\text { Extent of } \\
\text { Resection }\end{array}$} & \multicolumn{4}{|c|}{ Surgical Outcome } & \multirow{2}{*}{$\begin{array}{l}\text { Adjuvant } \\
\text { Therapy }\end{array}$} & \multirow{2}{*}{$\begin{array}{c}\text { Follow-Up } \\
\text { (mos) }\end{array}$} \\
\hline & & VD & AHPD & $\mathrm{DI}$ & Other & & \\
\hline 1 & PR & Improved & None & None & OMD (no change) & SRT & 41 \\
\hline 2 & STR & Deteriorated & No change & None & None & SRS & 34 \\
\hline 3 & STR & Improved & Deteriorated & None & None & SRT & 29 \\
\hline 4 & STR & Improved & Deteriorated & None & None & None & 22 \\
\hline 5 & NTR & No change & No change & Transient & None & None & 18 \\
\hline 6 & GTR & Improved & None & None & None & None & 17 \\
\hline 7 & STR & Improved & None & None & None & None & 17 \\
\hline 8 & GTR & Improved & None & None & None & None & 15 \\
\hline 9 & NTR & None & No change & No change & None & None & 14 \\
\hline 10 & GTR & No change & None & None & $\mathrm{CSDH}$ & None & 10 \\
\hline 11 & GTR & Improved & None & None & $\mathrm{CSDH}$ & None & 7 \\
\hline 12 & NTR & No change & Deteriorated & Permanent & None & SRT & 6 \\
\hline
\end{tabular}

AHPD = anterior hormonal pituitary dysfunction; PR = partial resection; SRS = stereotactic radiosurgery; SRT = stereotactic radiotherapy; STR = subtotal resection; $\mathrm{VD}=$ visual disturbance.

stimulating hormone [TSH], and luteinizing hormone/follicle-stimulating hormone [LH/FSH]) to 4 axes (GH, TSH, adrenocorticotropic hormone $[\mathrm{ACTH}]$, and $\mathrm{LH} / \mathrm{FSH})$; in Case 4, deficiencies in 1 axis $(\mathrm{GH})$ to 2 axes (GH and $\mathrm{ACTH})$; and in Case 12, deficiencies in 1 axis $(\mathrm{GH})$ to 4 axes (GH, TSH, ACTH, and LH/FSH). With regard to DI, one patient had permanent DI, and another had transient DI. No patient experienced postoperative hemorrhage, needed additional surgical treatment, or had postoperative CSF leakage. There was no perioperative mortality in this cohort. With respect to medium-term complications directly related to the surgery, 2 patients presented with a chronic subdural hematoma (CSDH) during follow-up and required surgical evacuation.

Four patients with residual tumor (3 pituitary adenomas and 1 craniopharyngioma) underwent postoperative radiotherapy. The other 4 patients with residual tumor were kept under clinical observation without adjuvant therapy. During follow-up, all residual tumors were well controlled without tumor recurrence that necessitated an additional surgical procedure. The average follow-up period was 19.2 months (range 6-41 months). The extent of resection, surgical outcomes, type of adjuvant therapies, and duration of follow-up are shown in Table 2.

\section{Discussion}

\section{Advantages of Simultaneous Combined TSA and TCA for Complicated Parasellar Lesions}

Recently, with advances in neuroendoscopy, many pituitary adenomas have been safely and totally resected via an eTSA. ${ }^{10,16,31}$ Moreover, extended TSA has been developed and is an effective treatment for other pathological lesions in the parasellar region, including craniopharyngiomas, tuberculum sellae meningiomas, and olfactory neu-

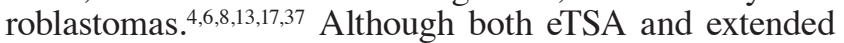
TSA can provide a direct corridor to parasellar lesions with low morbidity, total resection of tumors that extend far laterally beyond the ICA cannot be achieved with these approaches. Known to have a histologically benign nature, craniopharyngiomas have a tendency to adhere to surrounding vital structures, including the optic apparatus, the ICA and its branches, the pituitary stalk, and the hypothalamus. In addition, they often relapse rapidly after incomplete tumor resection. Residual pituitary adenomas can lead to postoperative hemorrhage, resulting in severe surgical sequelae unless total resection is achieved. Regardless of the tumor pathology, total tumor removal is the primary goal in optimizing tumor control. However, firm adherence to surrounding critical structures can be the main factor that impedes total tumor removal. Aggressive removal of this kind of tumor can result in postoperative surgical complications. As a result, the surgical strategy for parasellar tumors with fibrous consistency, tight adherence to the surrounding critical structures, or far-lateral extension beyond the ICA has been a matter of debate. With such a background, the simultaneous combined TSA-TCA was first reported in 1984 by Loyo et al. ${ }^{21}$ Since then, several types of combined TSAs-TCAs have been developed. ${ }^{1,5,11,15,18,19,23,24,27}$

The simultaneous combined TSA-TCA was first introduced to achieve maximal resection of pituitary adenomas that could be resected via either TSA or TCA alone. The addition of TCA to TSA improves visualization for complicated parasellar lesions and the surrounding neurovascular structures, contributing to better tumor resection. However, we are convinced that the greatest advantage of the simultaneous combined TSA-TCA is not only to achieve radical tumor resection, but also to provide safe tumor removal. As described above, complicated parasellar lesions interrupt the surgical view from the TSA, where critical structures can adhere to the superior and/or posterior aspects of the tumor. Since blind withdrawal of such tumors can result in severe surgical complications, it is necessary to identify the blind area of the TSA surgeon from another direction to provide safe tumor removal. If there is adhesion, it can be dissected via the TCA without any damage to the adjacent critical structures. A case il- 


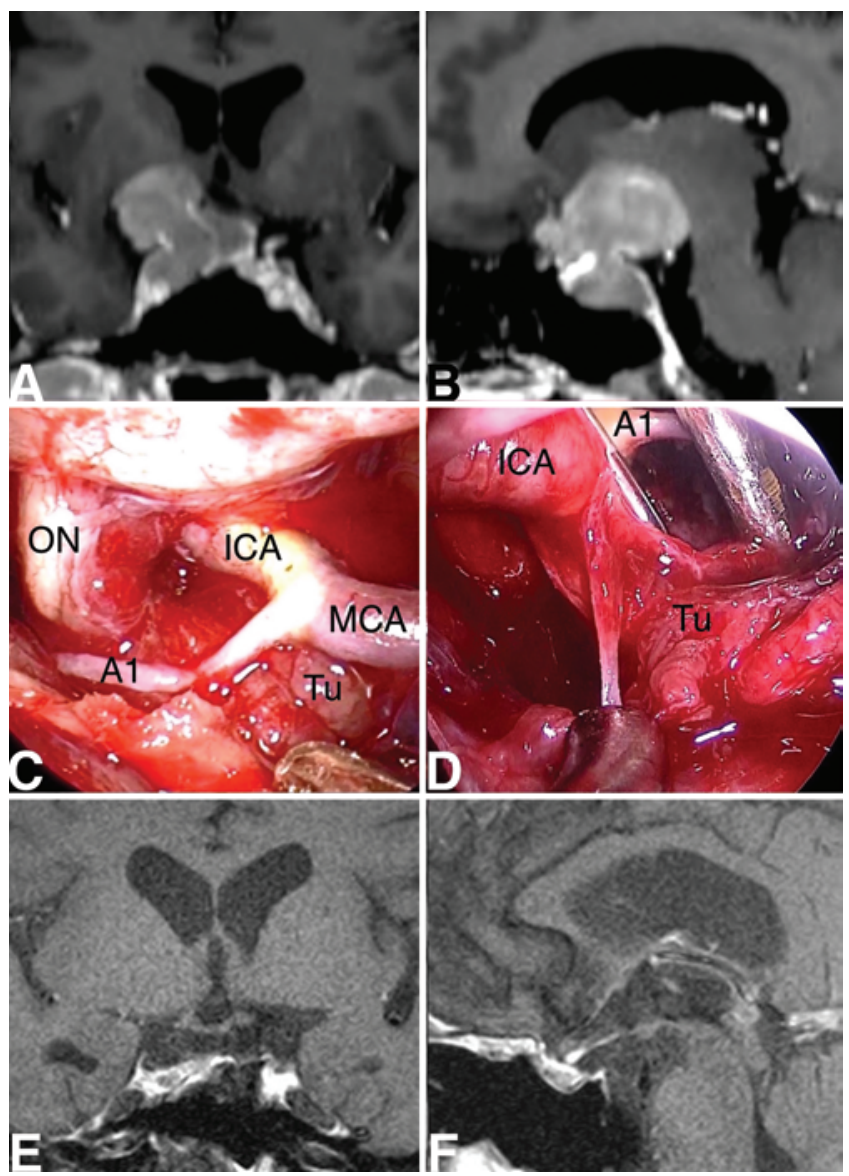

FIG. 3. Case 11. Pre- and postoperative imaging findings and intraoperative images. A and B: Preoperative coronal (A) and sagittal (B) Gdenhanced T1-weighted MR images showing that the pituitary tumor extends laterally beyond the right ICA and posteriorly beyond the dorsum sellae. C: Intraoperative image from the right SKA. The tumor adheres firmly to surrounding neurovascular structures, including the right optic nerve, right ICA, and right $A$, segment. $D$ : Intraoperative image from the TSA. The adhesion between the lateral aspect of the tumor and the right ICA cannot be detected by the TSA surgeon, so it is safely dissected via the SKA. E and F: Coronal $(E)$ and sagittal $(F)$ Gd-enhanced T1weighted MR images obtained 7 months after surgery, showing GTR of the pituitary tumor. MCA = middle cerebral artery. Figure is available in color online only.

lustrating this situation is presented in Fig. 3. Furthermore, it has often been reported that residual pituitary adenomas can induce postoperative hemorrhage with tumor swelling. ${ }^{29}$ Such intratumoral hemorrhage and tumor swelling, however, can also occur during tumor resection, which has never been previously reported. A case illustrating this situation is shown in Fig. 4. This phenomenon, the "angry" tumor (i.e., the tumor's appearance bears resemblance to the face of an angry person; Fig. 4D), can easily damage the adjacent critical neurovascular structures, resulting in postoperative morbidity, because neurosurgeons cannot be aware of this phenomenon when using TSA alone. Therefore, wide surgical exposure and cooperative manipulation from 2 directions in a simultaneous combined approach are important in preventing postoperative complications. As other options, a surgical team can perform these 2 approaches in sequence or by moving from one to the other

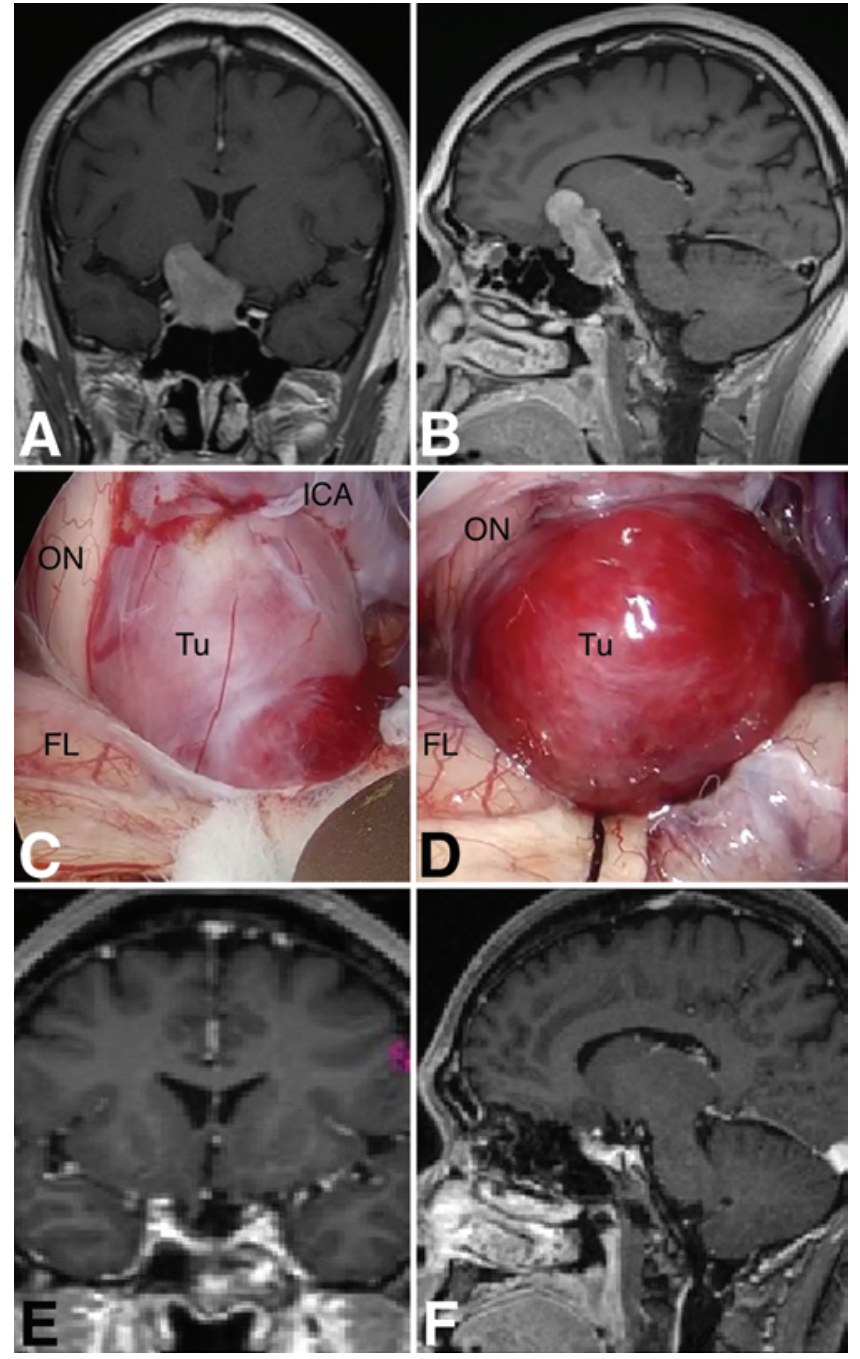

FIG. 4. Case 8. Pre- and postoperative imaging findings and intraoperative images. A and B: Preoperative coronal $(A)$ and sagittal $(B)$ Gd-enhanced T1-weighted MR showing that the pituitary tumor extends laterally beyond the right ICA, with invagination into the right frontal lobe. C: Intraoperative image from the right SKA. The tumor is located in the space between the right optic nerve and the right ICA. D: The tumor becomes hyperemic and swollen (an "angry" tumor), with progression of tumor debulking from the transsphenoidal approach. The compression of surrounding neurovascular structures is exacerbated by tumor swelling. E and F: Postoperative coronal $(E)$ and sagittal $(F) \mathrm{Gd}-$ enhanced T1-weighted MR images showing gross-total resection of the pituitary tumor. Figure is available in color online only.

alternately during a single general anesthesia session. Furthermore, staged surgeries with multiple general anesthetic agents are also another option. However, we are convinced that simultaneous cooperative manipulation from 2 directions can achieve safer and more effective tumor resection.

\section{Nontechnical Skills in Fully Endoscopic Combined TSA and SKA}

Cooperating from 2 corridors, team surgery is essential in fully endoscopic combined TSA-SKA. The eTSA and eSKA surgeons do not resect the tumor independently. Each surgeon is devoted to each operative monitor, so we consider that the presence of a third surgeon who observes 


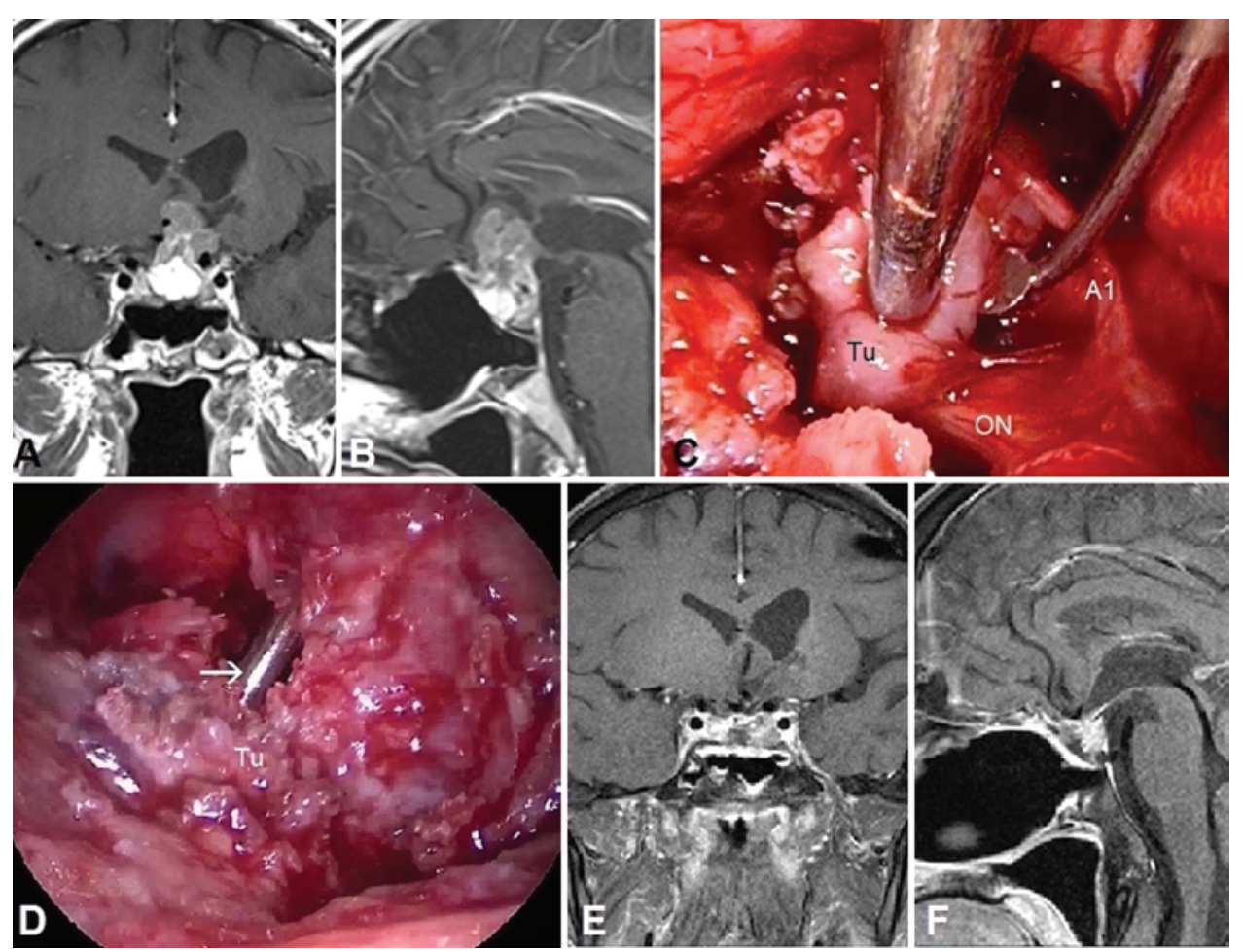

FIG. 5. Case 5. Pre- and postoperative imaging findings and intraoperative images. A and B: Preoperative coronal (A) and sagittal (B) Gd-enhanced T1-weighted MR images showing recurrent pituitary adenoma extending laterally beyond the left ICA and superiorly beyond the anterior communicating artery. C: Intraoperative image from the left SKA. The tumor adheres firmly to the left optic nerve and the left $A_{1}$ segment. The adhesion is safely dissected via this approach. $D$ : Intraoperative image from the eTSA obtained at the same time as the image in panel $C$. The surgical instrument for the SKA (white arrow), but not the adhesion between the superior part of the tumor and the $A_{1}$ segment, can be detected from the TSA. The tumor is resected via the TSA after the tumor adhesion to the $A_{1}$ segment is dissected from the SKA. E and F: Postoperative coronal $(E)$ and sagittal $(F) G d-$ enhanced T1-weighted MR images showing NTR of the recurrent tumor. Figure is available in color online only.

both surgical fields is important for effective cooperative manipulation from 2 directions. The third surgeon can provide the other 2 surgeons with precise instructions depending on the surgical situation, like a conductor of an orchestra. In some situations, the conductor may direct that the eSKA surgeon dissect the adhesion between the tumor and the optic nerve, and subsequently the eTSA surgeon to withdraw the tumor. The conductor can control the steps in the surgical procedure and facilitate the cooperative tumor removal from 2 directions. The strong leadership of the conductor and communication among the surgeons, which have been termed nontechnical skills in past articles, ${ }^{9,38}$ are also key success factors even in fully endoscopic combined TSA and SKA.

\section{The Indication for Simultaneous Combined TSA-TCA}

Eight patients who had previously undergone partial tumor resection via TSA and/or TCA due to firm adhesion of the tumor to surrounding critical neurovascular structures were included, supporting our indication for a simultaneous combined approach to achieve safer tumor resection. A strong adhesion is difficult to dissect via TSA alone, and it will result in perioperative hemorrhage or permanent sequelae if aggressive dissection is attempted. In reoperation of such cases, stronger adhesion to adjunct neurovascular structures should be anticipated, which makes GTR more difficult and makes the occurrence of postoperative complications more frequent. Therefore, we performed simultaneous combined TSA-TCA for such adherent tumors to achieve more effective and safer tumor resection from 2 surgical corridors when the residual adherent tumors had increased in size and had required additional surgical treatment (Fig. 5).

\section{Advantages of Fully Endoscopic Combined TSA-SKA}

As mentioned above, the simultaneous combined TSATCA approach has an excellent advantage for aggressive and safe tumor removal for complicated parasellar lesions. However, there are some disadvantages to this approach. First, 2 different operative fields, that is, TSA and TCA, are needed. If microscopes are used in both fields, the working space will be limited. Second, the conventional combined approach, TSA and a microscopic pterional, interhemispheric, or subfrontal approach, is highly invasive for patients, because this conventional TCA requires a relatively large craniotomy. However, the fully endoscopic combined TSA-SKA that we report here can overcome these disadvantages. The neuroendoscopic system in our combined approach is smaller than the microscopic system in the conventional combined approach, allocating a much more comfortable working space without interfering with the other surgical team. In addition, only a small keyhole craniotomy is needed in our minimally invasive technique, which is a much smaller burden to the patients. 
Moreover, there are also the potential advantages of the endoscopic technique, which contributes to a close and wide panoramic view of the surgical field with fine illumination, even in deep sites. It leads to clearer identification of critical structures and the arachnoid membrane, resulting in better surgical outcomes. ${ }^{16,22,31}$ The limitation of our combined approach, however, is the need for neurosurgeons to be competent to manipulate under an endoscope, which is considerably different from microsurgical technique. Furthermore, our combined approach requires 2 neuroendoscopic systems, which are not available at most institutions.

\section{The Features and Limitations of SKA}

The SKA is a minimally invasive surgical technique, appropriate to the parasellar region and anterior skull base. Many intracranial lesions, including pituitary adenomas, craniopharyngiomas, and meningiomas, are effectively resected via this approach., ${ }^{2,25,26,36}$ One of the greatest advantages of this approach is the exposure of the tumor portion extending far laterally beyond the ICA, where it cannot be accessed by eTSA alone. Moreover, an endoscope provides neurosurgeons with a close and wide panoramic view of the surgical field and can be used in the SKA. A much wider surgical view into the sella turcica, middle fossa, and prepontine cistern is available with an angled endoscope. With the combination of eSKA and eTSA, a wide surgical field ranging from the intra- and suprasellar regions to the far-lateral portion beyond the ICA and to the anterior/middle cranial fossa can be obtained. However, there are 2 limitations of the SKA. First, lesions with excessive invagination into the frontal lobe cannot be accessed via this approach. In such cases, simultaneous combined TSA and the transcortical cylinder approach can be an effective procedure. Second, parasellar lesions with bilateral extension beyond the ICAs cannot be totally removed via this approach. Simultaneous combined TSA and the conventional basal interhemispheric approach can achieve more aggressive resection for lesions with such a configuration in a single-stage procedure.

\section{Multimodality Therapy for Complicated Parasellar Lesions}

In past studies reporting on the simultaneous microscopic combined approach, $, 1,5,18,23$ the rate of GTR ranged from $24.1 \%$ to $44.4 \%$, which is equivalent to our outcomes. As is well known, one of the greatest risk factors for incomplete tumor resection is the presence of cavernous sinus invasion, especially circumferential encasement of the ICA, not only for TSA, but also for TCA.,10,16,22,39 In our cohort, 5 of 10 cases of pituitary adenomas were classified as Grade 4 of the Knosp-Steiner classification. ${ }^{14}$ Patients in all of these cases had excellent tumor control outcomes after resection followed by adjuvant radiotherapy or radiosurgery, similar to what has previously been reported..$^{10,16,20,22,39}$ Therefore, we are convinced that the multimodal approach of maximal surgical removal followed by adjuvant irradiation is the optimal treatment strategy for pituitary adenomas with extensive cavernous sinus invasion (Fig. 6). In addition, this strategy is effective for patients with residual craniopharyngiomas. Craniopharyngiomas often adhere firmly to the adjacent critical

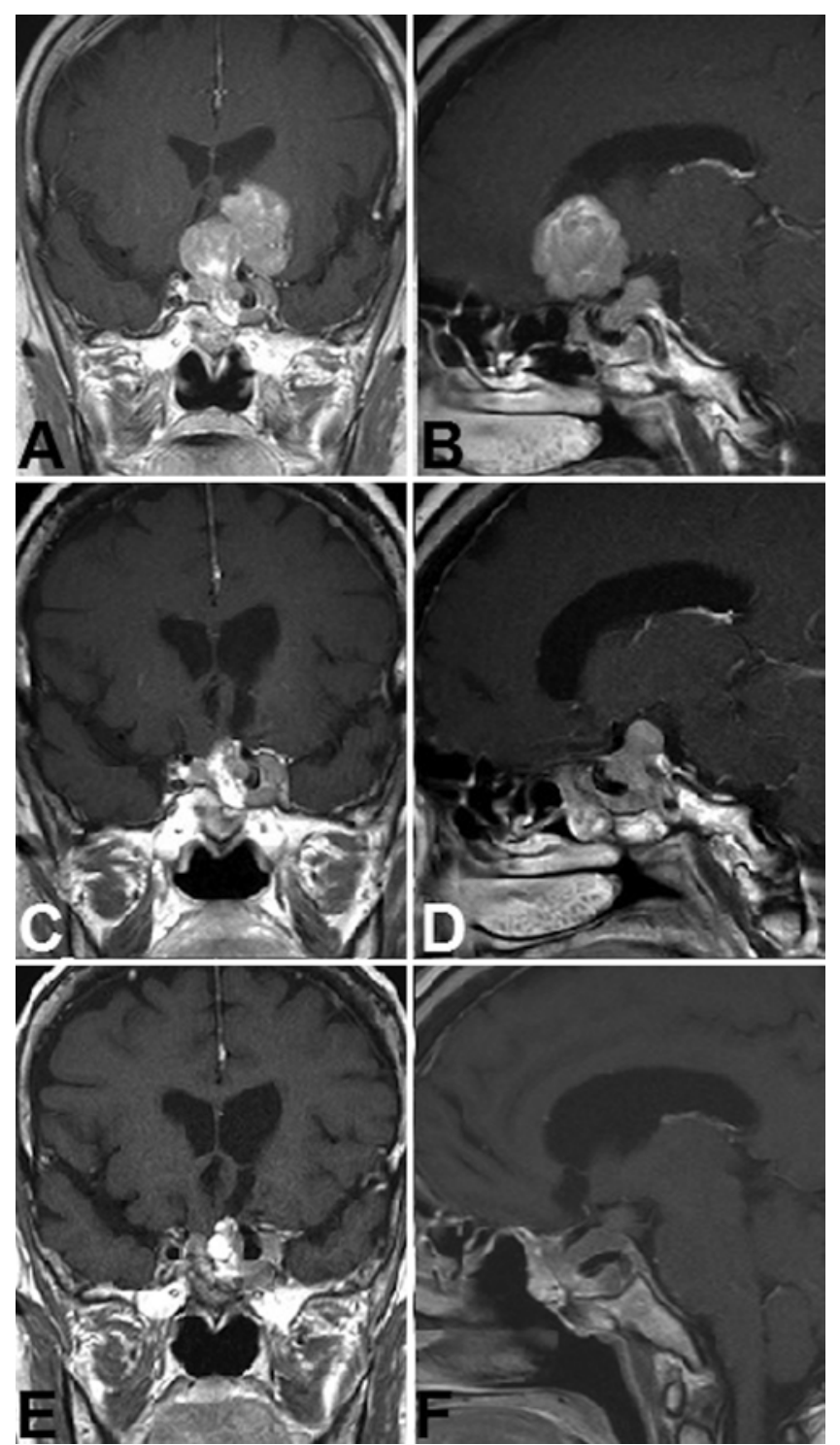

FIG. 6. Case 3. Pre- and postoperative imaging findings. A and B: Preoperative coronal (A) and sagittal (B) Gd-enhanced T1-weighted MR images showing recurrent pituitary tumor extending laterally beyond the left ICA. The tumor also infiltrates into the left cavernous sinus, and it encases the left intracavernous carotid artery. The tumor is subtotally resected via the fully endoscopic TSA-SKA, leaving the intracavernous part of the tumor. C and D: Postoperative coronal (C) and sagittal (D) Gd-enhanced T1-weighted MR images obtained 1 week after surgery, showing residual tumor in the left cavernous sinus. The patient underwent stereotactic radiotherapy for the left intracavernous residual tumor postoperatively. E and F: Coronal (E) and sagittal (F) Gd-enhanced T1weighted MR images obtained 9 months after stereotactic radiotherapy, showing the smaller size of the residual tumor.

neurovascular structures, so the point of strong adhesion to critical structures must remain to prevent postoperative complications. Previous studies have shown, however, that the tumor control rate of subtotal resection followed by adjuvant irradiation is equivalent to that of GTR in initial surgery. ${ }^{17,37}$ Residual craniopharyngiomas treated with adjuvant radiotherapy also had excellent tumor control in the present cohort. 


\section{Surgical Outcomes of Fully Endoscopic Combined TSA-SKA}

Postoperative visual improvement in the simultaneous microscopic combined approach ranged from $64 \%$ to $100 \%$, which is equivalent to the present outcome. The surgical complications of the simultaneous microscopic combined approach have also been reported, including postoperative permanent anterior hormonal pituitary dysfunction $(10 \%-72.7 \%)$, permanent DI $(6.9 \%-20 \%)$, CSF leakage $(0 \%-11.1 \%)$, and others $(8.3 \%-20 \%),{ }^{1,5,18,23}$ which were also comparable to the present results. Therefore, the outcomes of a fully endoscopic combined TSA-SKA showed noninferiority compared with the conventional microsurgical combined approach. However, there are some limitations to this study: the number of cases was small, and the individual patient follow-up was relatively short; thus, long-term outcomes of our combined approach cannot be assessed. Further accumulation of cases and longer follow-up periods are required to evaluate the true safety and efficacy of our combined approach.

Notably, 2 cases of postoperative CSDH were seen in the present cohort, which has never been reported as a postoperative complication of the simultaneous combined approach in past articles. However, there have been 2 case reports of CSDH after TSA..$^{30,35}$ Both patients were older than 70 years of age and had lumbar drainage placed for CSF rhinorrhea after the TSA; thus, advanced age, CSF rhinorrhea, and a lumbar drain were presumed to be the risk factors for CSDH after TSA. However, neither of the patients in our study who presented with postoperative CSDH had any of these risk factors. Moreover, our most recent study demonstrated that advanced age ( $>65$ years), high Evans' index, and preoperative hypopituitarism are the risk factors for CSDH after TSA. ${ }^{34}$ Although one of the 2 patients with postoperative CSDH was older than 65 years, no other risk factors were detected in the 2 patients. Frank et al. reported that 2 of 10 patients who had undergone extended TSA for craniopharyngioma developed CSDH postoperatively, ${ }^{8}$ which was a similar incidence to that seen in the present study. In fact, a larger volume of air retention is detected either after the extended TSA or after the simultaneous combined TSA-SKA compared with conventional TSA. Notable intraoperative CSF leakage, which results in postoperative pneumocephalus, may be an independent risk factor for postoperative CSDH. The small craniotomy in SKA may be related to this complication, compared with the larger craniotomy in conventional microscopic TCA. Studies involving more patients are needed to evaluate the actual risk factors for this complication.

\section{Conclusions}

A fully endoscopic combined TSA-SKA is an efficacious surgical procedure for a select group of patients with complex parasellar lesions. It is a new minimally invasive technique with excellent postoperative outcomes equivalent to the conventional microscopic combined approach. Safe and effective cooperative manipulation with 2 surgical corridors is available, and nontechnical skills that facilitate cooperation among the surgeons are also essen- tial. The main purpose of this procedure is not to achieve GTR, but to provide safe resection. Multimodality therapy, including postoperative adjuvant irradiation, is needed for lesions that cannot be totally resected even via this approach. Whatever the case, maximal surgical removal with minimal surgical morbidity is the optimal initial treatment. The appropriate surgical approach should be planned, depending on the configuration and consistency of each tumor.

\section{Acknowledgments}

We are grateful to Toshihiro Ogiwara, MD; Kenichi Wakabayashi, MD; Futoshi Kurimoto, MD; and Hirokuni Hashikata, $\mathrm{MD}$, for contributing clinical information about the patients.

\section{References}

1. Alleyne CH Jr, Barrow DL, Oyesiku NM: Combined transsphenoidal and pterional craniotomy approach to giant pituitary tumors. Surg Neurol 57:380-390, 2002

2. Berhouma M, Jacquesson T, Jouanneau E: The fully endoscopic supraorbital trans-eyebrow keyhole approach to the anterior and middle skull base. Acta Neurochir (Wien) 153:1949-1954, 2011

3. Cappabianca P, Cavallo LM, Esposito F, De Divitiis O, Messina A, De Divitiis E: Extended endoscopic endonasal approach to the midline skull base: the evolving role of transsphenoidal surgery. Adv Tech Stand Neurosurg 33:151-199, 2008

4. Cavallo LM, Frank G, Cappabianca P, Solari D, Mazzatenta D, Villa A, et al: The endoscopic endonasal approach for the management of craniopharyngiomas: a series of 103 patients. J Neurosurg 121:100-113, 2014

5. D'Ambrosio AL, Syed ON, Grobelny BT, Freda PU, Wardlaw S, Bruce JN: Simultaneous above and below approach to giant pituitary adenomas: surgical strategies and long-term follow-up. Pituitary 12:217-225, 2009

6. de Divitiis E, Esposito F, Cappabianca P, Cavallo LM, de Divitiis O, Esposito I: Endoscopic transnasal resection of anterior cranial fossa meningiomas. Neurosurg Focus 25(6):E8, 2008

7. de Paiva Neto MA, Vandergrift A, Fatemi N, Gorgulho AA, Desalles AA, Cohan P, et al: Endonasal transsphenoidal surgery and multimodality treatment for giant pituitary adenomas. Clin Endocrinol (Oxf) 72:512-519, 2010

8. Frank G, Pasquini E, Doglietto F, Mazzatenta D, Sciarretta V, Farneti G, et al: The endoscopic extended transsphenoidal approach for craniopharyngiomas. Neurosurgery 59 (1 Suppl 1):ONS75-ONS83, 2006

9. Gjeraa K, Spanager L, Konge L, Petersen RH, Østergaard D: Non-technical skills in minimally invasive surgery teams: a systematic review. Surg Endosc 30:5185-5199, 2016

10. Gondim JA, Almeida JP, Albuquerque LA, Gomes EF, Schops M: Giant pituitary adenomas: surgical outcomes of 50 cases operated on by the endonasal endoscopic approach. World Neurosurg 82:e281-e290, 2014

11. Greenfield JP, Leng LZ, Chaudhry U, Brown S, Anand VK, Souweidane MM, et al: Combined simultaneous endoscopic transsphenoidal and endoscopic transventricular resection of a giant pituitary macroadenoma. Minim Invasive Neurosurg 51:306-309, 2008

12. Honegger J, Ernemann U, Psaras T, Will B: Objective criteria for successful transsphenoidal removal of suprasellar nonfunctioning pituitary adenomas. A prospective study. Acta Neurochir (Wien) 149:21-29, 2007

13. Jho HD: Endoscopic transsphenoidal surgery. J Neurooncol 54:187-195, 2001 
14. Knosp E, Steiner E, Kitz K, Matula C: Pituitary adenomas with invasion of the cavernous sinus space: a magnetic resonance imaging classification compared with surgical findings. Neurosurgery 33:610-618, 1993

15. Koechlin NO, Simmen D, Briner HR, Reisch R: Combined transnasal and transcranial removal of a giant clival chordoma. J Neurol Surg Rep 75:e98-e102, 2014

16. Komotar RJ, Starke RM, Raper DM, Anand VK, Schwartz TH: Endoscopic endonasal compared with microscopic transsphenoidal and open transcranial resection of giant pituitary adenomas. Pituitary 15:150-159, 2012

17. Koutourousiou M, Gardner PA, Fernandez-Miranda JC, Tyler-Kabara EC, Wang EW, Snyderman CH: Endoscopic endonasal surgery for craniopharyngiomas: surgical outcome in 64 patients. J Neurosurg 119:1194-1207, 2013

18. Leung GK, Law HY, Hung KN, Fan YW, Lui WM: Combined simultaneous transcranial and transsphenoidal resection of large-to-giant pituitary adenomas. Acta Neurochir (Wien) 153:1401-1408, 2011

19. Leung GK, Yuen MM, Chow WS, Tse PY, Lui WM: An endoscopic modification of the simultaneous 'above and below' approach to large pituitary adenomas. Pituitary 15:237-241, 2012 (Erratum in Pituitary 15:242, 2012)

20. Losa M, Valle M, Mortini P, Franzin A, da Passano CF, Cenzato M, et al: Gamma Knife surgery for treatment of residual nonfunctioning pituitary adenomas after surgical debulking. J Neurosurg 100:438-444, 2004

21. Loyo M, Kleriga E, Mateos H, de Leo R, Delgado A: Combined supra-infrasellar approach for large pituitary tumors. Neurosurgery 14:485-488, 1984

22. Mortini P, Barzaghi R, Losa M, Boari N, Giovanelli M: Surgical treatment of giant pituitary adenomas: strategies and results in a series of 95 consecutive patients. Neurosurgery 60:993-1004, 2007

23. Nishioka H, Hara T, Usui M, Fukuhara N, Yamada S: Simultaneous combined supra-infrasellar approach for giant/ large multilobulated pituitary adenomas. World Neurosurg 77:533-539, 2012

24. Ojha BK, Husain M, Rastogi M, Chandra A, Chugh A, Husain N: Combined trans-sphenoidal and simultaneous transventricular-endoscopic decompression of a giant pituitary adenoma: case report. Acta Neurochir (Wien) 151:843-847, 2009

25. Reisch R, Marcus HJ, Kockro RA, Ulrich NH: The supraorbital keyhole approach: how I do it. Acta Neurochir (Wien) 157:979-983, 2015

26. Reisch R, Perneczky A: Ten-year experience with the supraorbital subfrontal approach through an eyebrow skin incision. Neurosurgery 57 (4 Suppl):242-255, 2005

27. Romano A, Chibbaro S, Marsella M, Oretti G, Spiriev T, Iaccarino $\mathrm{C}$, et al: Combined endoscopic transsphenoidaltransventricular approach for resection of a giant pituitary macroadenoma. World Neurosurg 74:161-164, 2010

28. Saito K, Kuwayama A, Yamamoto N, Sugita K: The transsphenoidal removal of nonfunctioning pituitary adenomas with suprasellar extensions: the open sella method and intentionally staged operation. Neurosurgery 36:668-676, 1995

29. Sinha S, Sharma BS: Giant pituitary adenomas-an enigma revisited. Microsurgical treatment strategies and outcome in a series of 250 patients. Br J Neurosurg 24:31-39, 2010

30. Sudhakar N, Vafidis JA: Subdural haematoma after transsphenoidal surgery. Br J Neurosurg 17:253-255, 2003

31. Tabaee A, Anand VK, Barrón Y, Hiltzik DH, Brown SM, Kacker A, et al: Endoscopic pituitary surgery: a systematic review and meta-analysis. J Neurosurg 111:545-554, 2009

32. Takakura K, Teramoto A: Management of huge pituitary adenomas. Acta Neurochir Suppl 65:13-15, 1996

33. Takeuchi K, Nagatani T, Wakabayashi T: How I do it: shoelace watertight dural closure in extended transsphenoidal surgery. Acta Neurochir (Wien) 157:2089-2092, 2015

34. Takeuchi K, Watanabe T, Nagatani T, Nagata Y, Chu J, Wakabayashi T: Incidence and risk factors of subdural hematoma after intraoperative cerebrospinal fluid leakage during the transsphenoidal approach. Pituitary 19:565-572, 2016

35. Tanaka Y, Kobayashi S, Hongo K, Tada T, Kakizawa Y: Chronic subdural hematoma after transsphenoidal surgery. $\mathbf{J}$ Clin Neurosci 9:323-325, 2002

36. Wilson DA, Duong H, Teo C, Kelly DF: The supraorbital endoscopic approach for tumors. World Neurosurg 82 (6 Suppl):S72-S80, 2014

37. Yang I, Sughrue ME, Rutkowski MJ, Kaur R, Ivan ME, Aranda D, et al: Craniopharyngioma: a comparison of tumor control with various treatment strategies. Neurosurg Focus 28(4):E5, 2010

38. Yule S, Flin R, Maran N, Rowley D, Youngson G, PatersonBrown S: Surgeons' non-technical skills in the operating room: reliability testing of the NOTSS behavior rating system. World J Surg 32:548-556, 2008

39. Zada G, Du R, Laws ER Jr: Defining the "edge of the envelope": patient selection in treating complex sellar-based neoplasms via transsphenoidal versus open craniotomy. J Neurosurg 114:286-300, 2011

\section{Disclosures}

The authors report no conflict of interest concerning the materials or methods used in this study or the findings specified in this paper.

\section{Author Contributions}

Conception and design: Nagata, Watanabe, Nagatani, Takeuchi. Acquisition of data: Nagata. Analysis and interpretation of data: Nagata. Drafting the article: Nagata. Reviewed submitted version of manuscript: Watanabe, Nagatani, Takeuchi, Chu, Wakabayashi.

\section{Supplemental Information \\ Previous Presentations}

Portions of this work were presented in poster form at the 7th World Congress for Endoscopic Surgery of the Skull Base and Brain, Chicago, Illinois, May 15-18, 2016.

\section{Correspondence}

Yuichi Nagata, Department of Neurosurgery, Nagoya University Graduate School of Medicine, 65 Tsurumai-cho, Showa-ku, Nagoya, Aichi 466-8550, Japan. email: you1ngta@gmail.com. 